\title{
Validation of the Hasford score in a demographic study in chronic granulocytic leukaemia
}

\author{
M J Thomas, J A E Irving, A L Lennard, S J Proctor, P R A Taylor, on behalf of the \\ Northern Region Haematology Group
}

\begin{abstract}
Chronic granulocytic leukaemia (CGL) is a rare disease. For most patients the only curative treatment (an allogeneic stem cell transplant) is not available. Survival varies between a few months to many years from diagnosis, and an accurate prediction of the duration of survival could help patients and clinicians make informed decisions about the many treatment options. In 1984, the Sokal score was introduced to stratify patients into risk groups. Recently, a new prognostic scoring system was proposed by Hasford and co-workers for interferon treated patients. We have analysed survival on an unselected population based cohort of patients using both the Hasford and the Sokal scores. In the group overall, neither score was predictive of survival, but in younger patients ( $<60$ years) treated with interferon, the Hasford score was highly predictive of survival, dividing patients into groups with a five year survival of $77 \%$ (45 patients) $v 33 \%$ (six patients) $v$ 14\% (31 patients) $(\mathbf{p}=0.01)$.

(F Clin Pathol 2001;54:491-493)
\end{abstract}

Keywords: chronic granulocytic leukaemia; interferon $\alpha$; prognosis; Hasford score; Sokal score

Chronic granulocytic leukaemia (CGL) is a clonal disease that results from an acquired genetic change in a haemopoietic stem cell. Clinically, it is a biphasic or triphasic disease that is usually diagnosed in chronic phase, with a median survival from diagnosis of around five years. After a variable period of time patients enter blastic crisis, sometimes via an accelerated phase, which leads to death usually within six months. The only curative treatment is an allogeneic stem cell transplant. However, this treatment option is only available to a small number of younger patients. The time to transformation varies from patient to patient and efforts have been made to predict the prognosis in individual patients by the use of prognostic scores based on clinical parameters recorded at diagnosis. The most widely recognised and used score was devised by Sokal et al in 1984, ${ }^{1}$ when standard treatment was with busulfan. More recently, most patients are initially treated with hydroxyurea and then given maintenance treatment with interferon $\alpha$ (IFN- $\alpha)$. In 1998, a new scoring system was developed by Hasford and co-workers (fig 1$)^{2}$; this scoring system was based on the outcome of patients treated with IFN- $\alpha$. Patients used to formulate and evaluate this score had been treated in 14 studies involving 12 institutions. In our study, we have compared the predictive value of the Hasford and Sokal scoring systems on a small but unselected population of patients from the database of the Northern Region Haematology Group.

\section{Methods}

Since 1 January 1988 members of the Northern Region Haematology Group (based on the former Northern Health Region of the UK, population 3.1 million) have prospectively registered all new cases of CGL centrally as part of the population adjusted clinical epidemiology (PACE) programme. ${ }^{34}$ We excluded patients with atypical chronic myeloid leukaemia and chronic myelomonocytic leukaemia. Full clinical details at presentation (including results of cytogenetic analysis) were collected. Treatment was at the discretion of the consultant in charge, but full details including outcome were recorded and patients are followed up annually until death. In addition to the registration from haematologists, the regional cytogenetics service provided annual details of all patients with Philadelphia chromosome $(\mathrm{t}(9 ; 22)$ translocation) diagnosed in the region that year. There is

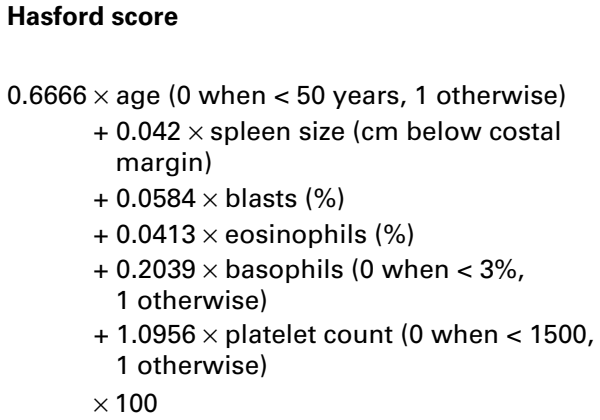

$\leq 780$ low risk group

$>780$ and $\leq 1480$ intermediate risk group

$>1480$ high risk group

\section{Sokal score}

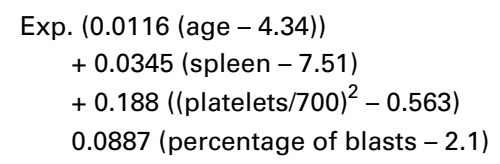

$<0.8$ good prognosis

0.8-1.2 moderate prognosis

$>1.2$ poor prognosis

Figure 1 How to calculate the Sokal and Hasford scores. 


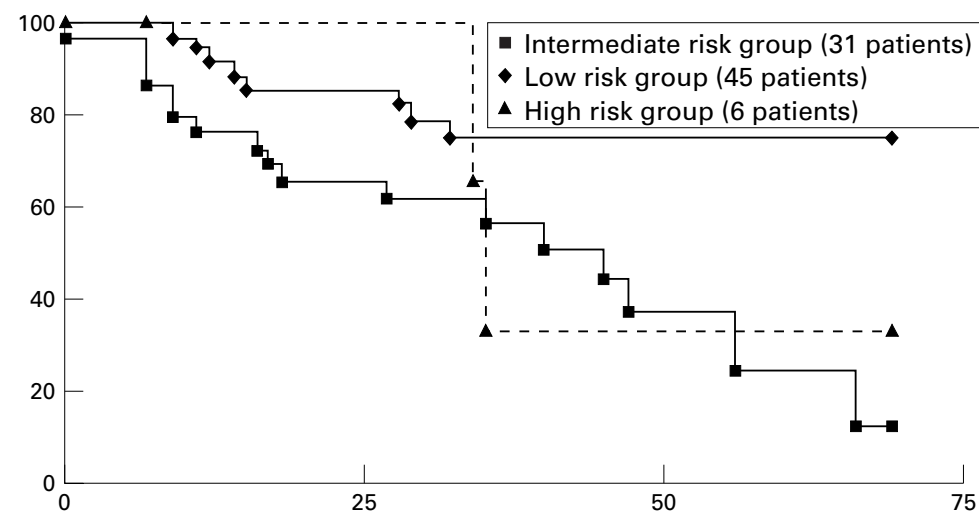

Figure 2 Actuarial overall survival in months by stratified Hasford score in patients $<60$ years old treated with interferon: patients receiving allogeneic transplants were censored at the time of transplantation, in accordance with the methodology in the original paper.

therefore a high degree of confidence that the database includes all patients diagnosed with CGL within our geographical area. As part of the PACE programme, a regional audit meeting took place in June 1993. Results of the first four years of data were discussed together with results of the Medical Research Council (MRC) CML $\mathrm{III}^{5}$ and other trials. At this meeting it was agreed that, in future, regional policy would be that all patients would be initially treated with hydroxyurea. Younger patients would be considered for an allogeneic transplant either with a matched sibling or matched unrelated donor, usually after a trial of IFN. Those without a donor or who were considered unsuitable for transplant would be given maintenance IFN to maximum tolerated dose. In our study, we have assessed the applicability of both the Sokal and Hasford scores to our population as a whole, and also to the subset of younger patients ( $<60$ years) who received treatment with IFN, because it is well recognised that some older patients are unable to tolerate IFN at optimal doses. In the analysis, patients receiving an allotransplant were censored at the time of allograft, as was done in the formulation of the original score. Eight patients had autologous transplantation in addition to IFN and were not censored. Survival was calculated using the KaplanMeier method $^{6}$ and curves were compared using the logrank test.

\section{Results}

There were 298 patients diagnosed with CGL in the northern region between 1 January 1988 and 1 May 1999; they had a median age of 59 years (range, 14-94), and there were 135 female and 163 male patients. Eighty two patients on the database fulfilled both the criteria of being under the age of 60 at diagnosis and presenting after 1 July 1993 when the audit recommended that they all be given a trial of treatment with IFN.

The Sokal and Hasford scores were applied to the whole cohort of patients and found to be uninformative (Sokal score, $\mathrm{p}=0.3$; Hasford score, $\mathrm{p}=0.2$, data not shown). We also applied both scores to younger patients treated before 1993 when neither hydroxyurea nor
IFN was standard treatment in our region and again they were unhelpful. The Hasford score was then applied to the unselected cohort of patients $<60$ years who had been treated with IFN; 21 of the 82 patients received an allograft, either matched sibling (nine patients, median time to transplant, 12 months; range, 5-29) or matched unrelated donor (12 patients; median time to transplant, 13 months; range, 9-57), and these were censored at the time of transplant. The Hasford score was found to indicate high risk in six patients $(7 \%)$, intermediate risk in $31(39 \%)$, and low risk in $45(55 \%)$, and overall survival of these groups is shown in fig 2 . The "high risk" group was small and had a survival of 33\% (95\% confidence interval (CI), 5-60\%; median, 35 months) but the Hasford score for "intermediate" and "low risk" separated patients into those with survivals of $14 \%$ (95\% CI, 3-25\%) at five years (median, 45 months) v 77\% (95\% CI, 69-85\%; median not reached; $\mathrm{p}=0.01$ ). On the same subset, although it did separate patients out, the Sokal score was less informative (survival: $78 \%$ low risk $v 34 \%$ intermediate risk $v 21 \%$ high risk; $p=0.05$ ). Given the small number of patients in the high risk group, we also analysed the results by combining the intermediate and high risk groups and analysing their survival compared with the low risk group. This gave the low risk group a survival of $77 \%$ (95\% CI, 85-95\%) compared with $19 \%$ for the combined intermediate and high risk groups (95\% CI, 8-30\%; $\mathrm{p}=0.006)$.

\section{Discussion}

The Hasford score was published in 1998 and as yet has not been validated in other populations. The main difference between this score and its predecessor, the Sokal score, is that the former assesses the impact of eosinophils and basophils on the differential white blood cell count at diagnosis (fig 1). The results of our study validate the Hasford scoring model. Although the Sokal score is still widely used, our study suggests that it is no longer the best method of reliably predicting length of survival for patients with CGL on IFN- $\alpha$. Because of small numbers ( $n=6 ; 7 \%$ of our total number of patients) we were unable to validate the applicability of Hasford's high risk score. Hasford and co-workers allocated nearly $15 \%$ of their original population to this high risk group, but the median age of their patients was greater than ours, which might account in some part for the discrepancy (median age $=49 v 45$ years, respectively).

Our low risk group had $77 \%$ survival, which compares with the original Hasford paper $(76 \%(\mathrm{SD}, 6 \%))$. However, our intermediate group gave poorer results than those predicted in the original paper, $14 \%(\mathrm{SD}, 11 \%) v 55 \%$ $(\mathrm{SD}, 6 \%)$ and we are unable to explain this.

Within the relatively small numbers of patients in this regional study, groups with significantly different median survival have been identified, demonstrating that the Hasford scoring system may be useful in identifying patients with a good prognosis using standard 
treatments. This may help these patients and their physicians to make better informed decisions about the adoption of alternative, higher risk, treatment options. It should also prove useful both in stratifying patients into risk groups and in the analysis of outcome in studies of newer, experimental drug regimens.

We would like to thank L Smith for secretarial help. Dr P Taylor is funded by a grant from the R \& D Department of Northern and Yorkshire Regional Health Authority. Members of the Northern Region Haematology Group at the time of this study Northern Region Haematology Group at the time of this study are: Dr M Abela, Dr N Browning, Dr P Carey, Dr R Cartner, Dr Goff, Dr P Hamilton, Dr A Hendrick, Dr A Iqbal, Dr F Jack, Dr G Jackson, Dr F Keenan, Dr J Kernahan, Dr P Kesteven, Dr A Lennard, Dr Z Maung, Dr I Neilly, Dr H O'Brien, Dr S O'Brien, Dr P Saunders, Dr G Summerfield, Dr H Tinegate, Dr J Wallis, Dr N West, Dr K Windebank, Dr P Williamson, Dr A Wood, Dr A Youart.
1 Sokal JE, Cox EB, Baccarani M, et al. Prognostic Sokal JE, Cox EB, Baccarani M, et al. Prognostic discrimination in "good-ris
mia. Blood 1984;86:726-36.

mia. Blood 1984;86:726-36.
2 Hasford J, Pfirmann M, Hehlmann R, et al. A new prognostic score for survival of patients with chronic myeloid leukaemia treated with interferon alfa. 7 Natl Cancer Inst 1998;90:850-8.

3 Charlton B, Taylor PRA, Proctor SJ. The PACE (population-adjusted clinical epidemiology) strategy: a new approach to multicentre clinical research. $O F \mathrm{Med}$ 1997; 90:147-51.

4 Taylor PRA, Angus B, Owen JP, et al. Hodgkin's disease: a population-adjusted clinical epidemiology study (PACE) of management at presentation. Qf Med 1998;91:131-9.

5 Allan NC, Richards SM, Shepherd PCA, on behalf of the UK Medical Research Council's Working Party for Therapeutic Trials in Adult Leukaemia. UK Medical Research peutic Trials in Adult Leukaemia. UK Medical Research Council randin of cytogenetic response. Lancet 1995;345:1392-7.

6 Kaplan EL, Meier P. Nonparametric estimation from incomplete observations. Fournal of the American Scientific Association 1958;53:457-81.

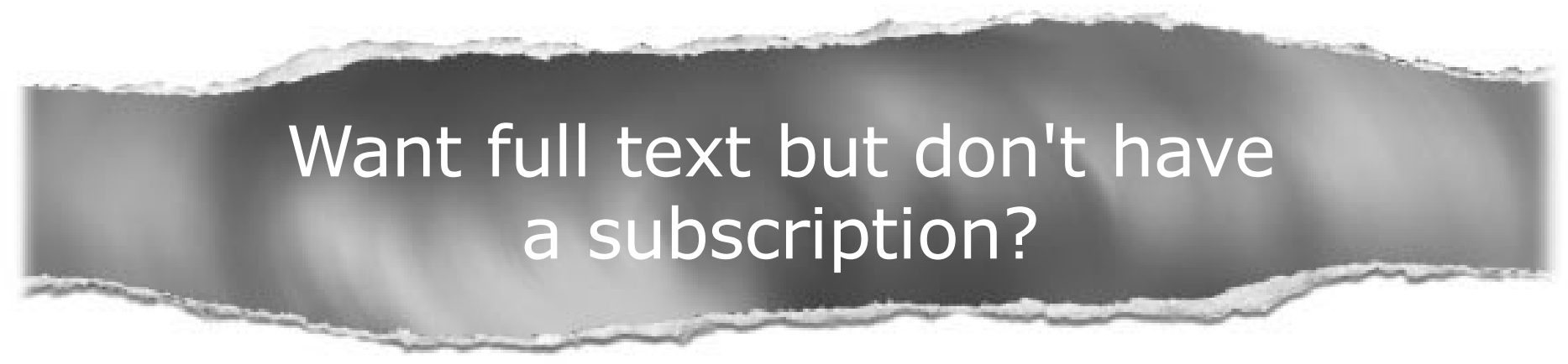

\section{Pay per view}

For just $\$ 8$ you can purchase the full text of individual articles using our secure online ordering service. You will have access to the full text of the relevant article for 48 hours during which time you may download and print the pdf file for personal use.

\section{www.jclinpath.com}

